Thorax, 1980, 35, 905-906

\title{
Recurrent major haemoptysis: progression to pneumonectomy
}

\author{
WILLIAM A BAUMGARTNER AND JAMES B D MARK \\ From the Department of Surgery, Division of Thoracic Surgery, Stanford University School of \\ Medicine, California, USA
}

ABSTRACT A 50-year-old white man with recurrent major haemoptysis is described. The main problem in management was in detecting the aetiology and source of the haemoptysis. Rigid bronchoscopy is essential in the evaluation and treatment of patients with massive haemoptysis, and once the site of bleeding has been established, pulmonary resection offers the best chance of survival. The operation performed is usually lobectomy, although pneumonectomy, as with our patient, may be necessary.

\section{Case report}

This 50-year-old white man (JA) presented to Stanford University Medical Center Emergency Room with the chief complaint of haemoptysis, having coughed up approximately $150 \mathrm{ml}$ of bright red blood in the previous two hours.

The history of the present illness began six years earlier when he was seen with what was considered to be epistaxis. After lesser measures failed, control of the bleeding was accomplished by ligation of the right internal maxillary artery. Two years later he underwent emergency bronchoscopy for massive haemoptysis. Chest radiographs were normal during this two-year interval. While still in the hospital on that occasion he experienced recurrent haemoptysis $(800 \mathrm{ml})$, again requiring emergency laryngoscopy, nasopharyngoscopy, and bronchoscopy. It could not be determined whether the site of bleeding was in the upper or lower respiratory tract. Posterior nasal packs appeared to control the haemorrhage during that episode. Because of the uncertainty of the specific location he underwent bilateral carotid arteriograms which were normal. Chest radiographs showed bilateral lower lobe infiltrates which cleared completely after each acute bleeding episode.

Two years later he again presented with haemoptysis (200-300 ml) which occurred on two separate occasions. The first episode was controlled with posterior nasal packs and the second

Address for reprint requests: Professor James Mark, Division of Thoracic Surgery, Stanford University School of Medicine, California 94305, USA. required bronchoscopy. Upon recovery he was transferred to Stanford University Medical Center. Nasopharyngoscopy was carried out and biopsy of a nasopharyngeal ulcer was performed (pathology: non-caseating granulomata). Bronchoscopy revealed old blood and clots in the lower lobe bronchi predominantly on the left side. Urinalysis on admission revealed no microscopic haematuria. Sputum cytology and skin tests were non-diagnostic. Further investigations included pulmonary and bronchial arteriograms and a thoracic aortogram which were normal.

One month after discharge the patient returned with haemoptysis $(300 \mathrm{ml})$. Bronchoscopy again revealed clots predominantly in the left bronchial tree. Bilateral external and internal carotid arteriograms were repeated. Occlusion of the sphenopalatine branch of the right external carotid artery was noted but no other abnormalities. A detailed haematological investigation showed no defects in the coagulation pathways.

The patient was well until this present admission. During diagnostic bronchoscopy, carried out under general anaesthesia, he bled massively from the left tracheobronchial tree. This was the first time a definite lateralisation of the bleeding source could be established. A Robertshaw double lumen endotracheal tube was inserted to protect the right lung. As we realised that left pneumonectomy might well be necessary, an attempt was made to embolise the bronchial arteries with Gel-foam in order to control the bleeding. After embolisation of two bronchial arteries the patient was nursed in the intensive care unit with the endotracheal tube in place, and 
he remained clinically stable. Two days later he again bled massively through the endotracheal tube and so an emergency left pneumonectomy was performed. The procedure was complicated by cardiac arrest and a pneumothorax on the right side. However, his postoperative course was uneventful and he was discharged 10 days after the operation.

Careful gross and microscopic examination of the lung revealed no site of bleeding or aetiology for the recurrent haemoptysis. He has not bled in the ensuing 22 months.

\section{Discussion}

The amount of bleeding in "massive haemoptysis" has been stated to be between 200 and $600 \mathrm{ml}$ over a 24-hour period. Most reviews record that massive haemoptysis is associated with a $50 \%$ mortality in those patients who are managed without operation. ${ }^{1}$ The mortality rate, however, also depends upon the degree of flooding of the tracheobronchial tree.

The major problem in management of this patient was determining the aetiology and source of his haemoptysis. Operation was not considered initially because it was unclear until the final bronchoscopy that he was indeed bleeding from his tracheobronchial tree and, more specifically, his left lung.

Diagnostic bronchoscopy should be performed in all patients with massive haemoptysis in order to localise or at least lateralise the site of bleeding. ${ }^{2}$ This procedure is essential even if there is a specific lesion on the chest radiograph. It is necessary to compile all available information in preparation for operation which may be necessary under urgent circumstances. Nasopharyngoscopy and laryngoscopy are frequently helpful in ruling out the upper respiratory tract as the source of bleeding. Bronchoscopy can also be therapeutic and life-saving as seen in our patient.

If time permits, further evaluation including sputum cytology, cultures, and skin tests should be performed. If there is no radiographic evidence of a specific lesion and bronchoscopy is unrevealing, further invasive studies such as pulmonary, carotid, and bronchial arteriography should be carried out. ${ }^{3}$ Bronchography can be helpful after sufficient time has elapsed to allow for clearing of blood from the bronchial tree.

Death from haemoptysis is generally the result of suffocation rather than exsanguination. Rigid bronchoscopy is essential in the evaluation and treatment of patients with massive haemoptysis. If a bleeding point or segment is identified, sponges can be used to tamponade the area. Fogarty balloon catheters can be placed directly into the bronchus to tamponade the bleeding and prevent spillover into normally aerated areas of the lungs. A double lumen endotracheal tube can be placed to prevent contralateral aspiration of blood. The placement of this tube requires expertise but when it is in the proper position it allows the anaesthetist to ventilate the uninvolved lung and aspirate the bleeding site.

The most common cause of massive haemoptysis is tuberculosis, followed by bronchiectasis, lung abscess, carcinoma, and other less common entities such as Wegener's granulomatosis, actinomycosis and sarcoma. Occasionally no cause can be found for the haemoptysis.

Once the site of bleeding in patients with massive haemoptysis has been localised pulmonary resection offers the patient the best chance of survival. ${ }^{45}$ The operation performed is usually lobectomy, although pneumonectomy may be necessary. Reported operative mortality is approximately $17 \%$ as compared to the $50-70 \%$ mortality from non-surgical management. This entity remains one of the most challenging problems in thoracic surgical practice.

\section{References}

1 Garzon AA, Gourin A. Operative treatment of massive hemoptysis. Ann Thorac Surg 1974; 18: 52-60.

2 Simmons DH, Wolfe JD. Hemoptysis: diagnosis and management. West $J$ Med 1977; 127:383-90. 근

3 Bookstein JJ, Davis GB, Higgins CB, James WS, Kalafer ME, Moser KM. The role $N^{\circ}$ of bronchial arteriography and therapeutic embolization in hemoptysis. Chest 1977; 72: 658-61.

4 Beall AC, Guinn CA, Mattox KL, McCollum WB. Immediate operative treatment for massive hemoptysis. Chest 1975; 67:152-5.

5 Garzon AA, Gourin A. Surgical management of $\stackrel{\mathscr{D}}{+}$ massive hemoptysis. Ann Surg 1978; 187:267-71. 